\title{
Liderazgos y estructura empresarial solidaria en la pesca artesanal colombiana
}

\author{
Jorge Iván Luque-Berkowitz ${ }^{\star}$, Sohely Rúa-Castañeda ${ }^{\star *}$
}

* Magíster (c) en Desarrollo Regional, Centro de Investigación en Alimentación y Desarrollo, Sonora, México.

Correo electrónico:

ivann_ok@hotmail.com

* Maestría en Educación y Desarrollo Humano, Universidad de Manizales. Especialista en Educación solidaria, Instituto de Economía Social y

Cooperativismo, Universidad

Cooperativa de Colombia, Bogotá, Colombia.

Correo electrónico:

sohely.rua@ucc.edu.co

Recibido: 17 de marzo del 2014

Aprobado: 8 de mayo del 2014

Cómo citar este artículo: Luque-Berkowitz, J. I. y Rúa-Castañeda, S. (2014). Liderazgos y estructura empresarial solidaria en la pesca artesanal colombiana. Cooperativismo \& Desarrollo, 104(22), 9-20. doi: http://dx.doi. org/10.16925/co.v22i104.969

\begin{abstract}
Resumen
El estudio presenta un panorama del liderazgo en las organizaciones solidarias de pesca artesanal colombianas, así como de las problemáticas que enfrentan para su fortalecimiento socioempresarial. El análisis de enfoque cualitativo se fundamenta en entrevistas semiestructuradas realizadas a líderes de diversas entidades y de formas asociativas de pesca artesanal en particular. Los hallazgos indican que el liderazgo solidario afronta un dilema ético y operativo en la fórmula democrática de la gestión y el avance en estructura empresarial; en el caso de la pesca artesanal, específicamente, también se enfrenta a un entorno socio-ambiental que reta la definición de su posición competitiva. Se concluye en la necesidad de generar una visión empresarial equilibrada que dé sostenibilidad a la actividad económica acorde con los retos del contexto, y que la vivencia de los principios cooperativos armonicen con esta búsqueda, lo cual significa reconocer los intereses personales y colectivos, los niveles diferenciados de participación y el desarrollo de capacidades técnico-operativas para gestar la empresa asociativa.
\end{abstract}

Palabras clave: cooperativas pesqueras, cooperativismo, economía solidaria, empresa solidaria, liderazgo cooperativo.

\section{Leadership and Solidarity Business Structure in Colombian Artisanal Fishing}

\section{Abstract}

This study presents an overview of the leadership at Colombian artisanal fishing solidarity organizations, along with the problems they face in their social and entrepreneurial strengthening. The qualitative approach analysis is based on semi-structured interviews with leaders from diverse organizations and associative forms of artisanal fishing in particular. The findings indicate that solidarity leaderships face an ethical and operative dilemma with respect to the democratic management formula and progress in their entrepreneurial structure. In the specific case of artisanal fishing, the socio-environmental context poses a challenge to its competitive position. The conclusion is that there is a need to generate a balanced entrepreneurial vision that would provide sustainability for this economic activity in accordance with the challenges in the context, and that cooperative principles must be applied in harmony with this effort, which signifies recognizing personal and collective interests, differentiated levels of participation and the development of technical-operative capacities for managing associative enterprises.

Keywords: fishing cooperatives, cooperativism, solidarity economy, solidarity enterprise, cooperative leadership.

\section{Lideranças e estrutura empresarial solidária na pesca artesanal colombiana}

\section{Resumo}

O estudo apresenta um panorama da liderança nas organizações solidárias de pesca artesanal colombianas, assim como das problemáticas que enfrentam para seu fortalecimento socioempresarial. A análise de enfoque qualitativo fundamenta-se em entrevistas semiestruturadas realizadas com líderes de diversas entidades e de formas associativas de pesca artesanal em particular. As descobertas indicam que a liderança solidária enfrenta um dilema ético e operativo na fórmula democrática da gestão e do avanço em estrutura empresarial; no caso da pesca artesanal, especificamente, também enfrenta um ambiente socioambiental que desafia a definição de sua posição competitiva. Conclui-se sobre a necessidade de gerar uma visão empresarial equilibrada que dê sustentabilidade para a atividade econômica de acordo com os desafios do contexto, e que a vivência dos princípios cooperativos harmonize com essa busca, o qual significa reconhecer os interesses pessoais e coletivos, os níveis diferenciados de participação e do desenvolvimento de capacidades técnico-operacionais para gestar a empresa associativa.

Palavras-chave: cooperativas pesqueiras, cooperativismo, economia solidária, empresa solidária, liderança cooperativa. 


\section{Introducción}

Liderazgo empresarial, motivacional y asociativo son algunos de los perfiles requeridos en una organización solidaria para llevar a cabo su objeto social. De la misma manera, la estructura empresarial asociativa y la actividad económica como tal requieren ir en sincronía para alcanzar los objetivos sociales y los empresariales. Este estudio aborda las dimensiones anotadas, liderazgos y estructuras organizacionales, en particular analizadas en el sector de la pesca artesanal colombiana, con el fin de dimensionar los retos a los que se enfrentan las asociaciones de diverso tipo que están vinculadas a la actividad pesquera.

Este trabajo, de corte cualitativo-descriptivo, tuvo como principal instrumento de indagación la entrevista a líderes de organizaciones solidarias a partir de unas premisas investigativas: las organizaciones empresariales requieren diferentes tipos de liderazgo para ser sostenibles; la competitividad está asociada a una estructura empresarial pertinente para el entorno.

El trabajo investigativo señala la necesidad de profundizar en cómo resolver las tensiones entre los objetivos sociales y empresariales, que el contexto económico actual genera. El modelo de gestión solidario y cooperativo centrado en promover la dinámica democrática dentro de las organizaciones precisa desarrollos teóricos en el sentido empresarial sobre cómo dinamizar la producción y llevar a cabo las actividades ligadas a la actividad económica sin contradecir la filosofía solidaria.

El documento se presenta estructurado en dos segmentos: una mirada general a los temas de liderazgos en esquemas empresariales y también en cuanto a la estructura organizacional, vistos desde el lente de la gestión empresarial solidaria. En la segunda sección, se analizan en particular los aspectos antes mencionados estudiados en las cooperativas de pesca artesanal del Caribe colombiano, en Santa Marta, Taganga y Pueblo Viejo.

\section{Gestión empresarial solidaria: entre lo social y los negocios}

Las asociaciones, cooperativas y otras organizaciones de la economía solidaria (oes) surgen como alternativas de producción y gestión al modelo económico capitalista imperante. En medio de una gama amplia de formas asociativas, existen estructuras en las cuales los asociados son, simultáneamente, los encargados de llevar a cabo la producción o la prestación del servicio objeto de la entidad jurídica.

De estas últimas formas organizacionales trata este estudio, es decir, en aquellas en las que el trabajo asociado genera la actividad económica, aunque su figura jurídica no sea una cooperativa de este tipo. En esta doble condición, de asociado y gestor, subyace una tensión que impone el liderazgo y la estructura solidaria a su propia gestión empresarial, que puede llevar al deterioro de su posición competitiva. Liderazgos naturales de tipo motivacional y con debilidades gerenciales no logran contribuir al impulso organizacional, y las estructuras empresariales no avanzan hacia nuevos niveles de complejidad, limitando la capacidad productiva, comercial y financiera que demanda actuar en el mercado.

Además, existen otros conflictos como la informalidad con que operan, la simplicidad organizativa y el exceso de democracia que, como Sommers (2006) señala, son originados por la falta de capital y de liderazgo con suficiente formación política y académica (valores y costumbres), factores que limitan el avance en un enfoque de eficiencia y productividad.

\section{Liderazgo y dirección}

La literatura de la economía solidaria y el cooperativismo propone una dirección organizacional compartida de manera democrática. Asimismo, exalta la búsqueda del bienestar de los agremiados, que debe ser un resultado tan importante como la generación de excedentes económicos. Sin embargo, es común encontrar que los directivos no tienen experiencia administrativa y gerencial (Segura-Aguilar, Rodríguez-Van Dyck y Weaver, 2009), por lo cual los objetivos empresariales no se alcanzan; esto, a su vez, genera efectos negativos en lo social, ya que los asociados se desmotivan al no encontrar respuesta adecuada a sus necesidades, y por ende se debilita la dinámica colectiva, dejando espacio para que individualmente algunas personas tomen beneficios para sí mismos en detrimento del bien común.

De otro lado, en la actualidad, el modelo autogestionario en comunidades con bajos niveles culturales y educativos se convierte en retos de inserción empresarial en mercados altamente competidos y enfrentados a problemáticas globales; en el caso que ocupa a este estudio, las de tipo ambiental, que demandan incorporar innovación tecnológica y social, capital y competencias del equipo humano. 
Algunos estudios promueven la participación de agentes externos (Arcas y Hernández, 2013; Karsten, 2009).Tal propuesta, contraria a la autogestión, se sustenta en la precaria situación técnica y tecnológica en la que se desenvuelven algunas organizaciones solidarias, por lo que se considera que resulta conveniente la intervención de un agente preparado que, momentáneamente, lleve la dirección. Esto no deja de generar un conflicto o tensión entre los objetivos socio-democráticos y el fortalecimiento empresarial, puesto que algunos pueden interpretar que se está desdibujando el modelo de gestión cooperativo y solidario.

El lado opuesto de esta discusión surge cuando se revisan experiencias asociativas sólidas en lo empresarial, ya que se plantean cuestionamientos a su modelo solidario en cuanto se privilegian los resultados económicos y se generan estructuras duales: por un lado, la empresarial, con la lógica capitalista, y otra social representada en el gobierno cooperativo que tiene injerencia al final del ejercicio económico en la asignación de los excedentes, pero no en la vida cotidiana de la empresa, lo cual deriva en una mirada de cliente respecto al asociado.

En la discusión planteada, el debate conduce a la necesidad de pensar el modelo empresarial como tal desde la filosofía solidaria; las prácticas democráticas por sí mismas no garantizan que se estén tomando decisiones adecuadas; socios, directores, gerentes y empleados, todos, necesitan adquirir competencias para la toma de decisiones empresariales (Segura-Aguilar et al., 2009).

Es importante señalar que algunos ejemplos de liderazgo en cooperativas exitosas están relacionados con una visión empresarial que es manejada de manera eficiente desde los puestos directivos. Esto se ve reflejado principalmente en algunas cooperativas financieras que, sin perder la conciencia social, han podido aprovechar algunos puntos de teorías administrativas recientes. Estos ejemplos señalan un cambio de enfoque: organizaciones centradas en la productividad, en contraste con estructuras que orientan su trabajo en el sentido social de la producción (Fiedler, 1967). Por supuesto, la necesidad del equilibrio salta a la vista, entre una organización que incorpora teorías y metodologías de gestión empresarial, y la filosofía asociativa, que mantiene el enfoque solidario como práctica y objetivo por alcanzar.

Algunas veces se elige al líder carismático (Weber, 1920) por la percepción de capacidades extraordinarias, lo que puede resultar eficiente para la integración de la cultura organizacional; pero también puede fundamentarse en lógicas irracionales (Lussier y Achua, 2008) que no prevén el futuro de la organización. Es común que estos directivos no tengan experiencia en administrar o en dirigir una organización. En este punto surge la cuestión sobre el papel del líder; puede ser más sencillo para quienes están asociados elegir a sus representantes por empatía, sin tener en cuenta la capacidad de llevar a cabo retos como la integración y el crecimiento empresarial. El primer aspecto de tipo motivacional es clave, pero en cuanto al segundo, se requieren competencias, por ejemplo la capacidad para tomar decisiones empresariales.

Desempeñar funciones gerenciales requiere conocimientos y habilidades (Segura-Aguilar et al., 2009). Los estilos de liderazgo son variados y se complementan con las características de los seguidores, las cuales constituyen el punto clave que desarrolla las acciones estratégicas, y que no siempre surgen de los puestos directivos pero que generalmente se adjudican a las capacidades de hombres y mujeres líderes. Pero no todo depende del carisma y estilo de dirección de los líderes de las empresas solidarias; el desarrollo de la estructura empresarial como tal es fundamental.

Aunado a lo anterior, algunos estudios (Kalogeras, Pennings, Benos y Doumpos, 2013) indican que el sentido de pertenencia y la identidad de los socios hacia la organización son esenciales para el fortalecimiento de esta. El compromiso refuerza a la cooperativa como tal, aunque no siempre se logra este compromiso en todos los miembros; estos aspectos deben ser tenidos en cuenta por la dinámica organizativa. Castañeda, Guido y Medina (2012) señalan que:

Aparentemente este interés colectivo es el resultado en gran medida de la implementación de mecanismos de regulación efectivos de la conducta de dirigentes y socios que afectaban negativamente el beneficio común. La existencia de reglamentos [...] minimizan los conflictos internos y evitan rupturas en la organización (p. 85).

En estas cooperativas, puede entenderse como un liderazgo cooperativo (Fortier, 2011) que surge del interior como resultado de compartir el poder, buscando los mismos objetivos. Incluso el director puede provenir de fuera de la comunidad, como propuesta de acompañamiento o coaching (Whitmore, 1992) y adoptar los objetivos de la organización como propios y aplicar sus capacidades personales, para cumplir los 
objetivos comunes. Desde este enfoque, el manejo del equilibrio entre la democracia y los lineamientos directivos objetivos sigue siendo una responsabilidad compartida entre el líder y los liderados, como dos partes que desarrollan su rol en interdependencia, y a la vez con suficiente libertad que les permita avanzar en sus planes orientados a la salud financiera, el mejoramiento comunitario y la responsabilidad social.

De igual manera, es necesario encontrar a los individuos adecuados para llevar tal proceso. Es evidente que se debe formar un grupo directivo en el cual se identifiquen las capacidades para un liderazgo centrado en las tareas (Fiedler, 1967), pero que a la vez sepa convencer a sus seguidores y los lleve a comprometerse con los objetivos.

\section{Modelo de gestión solidario}

Mejorar la calidad de vida de los miembros de las organizaciones de economía solidaria puede ser un objetivo común; sin embargo, los medios para lograrlo cambian en diferentes latitudes. Pensar las organizaciones solidarias en el sentido de que son estructuras cuyo propósito no termina ni empieza con la obtención de la ganancia económica, hace necesario iniciar la discusión acerca de la clasificación como entidades sin ánimo de lucro. Esta categorización oculta el interés económico legítimo de la empresa asociativa para generar riqueza y distribuirla entre sus miembros y con la sociedad en general; por tanto, es preciso diferenciarla de las economías de las donaciones, donde la bandera de la gratuidad es necesaria. La implicación de este ocultamiento del interés económico de tipo asociativo hace suponer que la gestión de las organizaciones de esta naturaleza solo demanda procesos democráticos para su desarrollo, descartando la dimensión propiamente empresarial. En este sentido se concuerda con Castañeda et al. (2012):

Una cooperativa exitosa es una organización productiva en donde el interés colectivo predomina sobre el individual, cuenta con capacidad de generar ingresos económicos, mantiene la cohesión social, genera empleos locales, fortalece la identidad y alcanza niveles de eficiencia y productividad que impactan el bienestar del socio y de su comunidad, al mismo tiempo que desarrollan y fortalecen a la propia organización (p. 21).

Esto traduce en que de la forma como se vivan los valores cooperativos y solidarios, se "modificará la dirección de los esfuerzos y estrategias, así como los resultados que se originen, lo cual repercutirá en el desarrollo cooperativo" (Mercader, 2013); y de otro lado, las estructuras asociativas que cuentan con excedentes, fundamentalmente como resultado de la comercialización directa en mercados atractivos, generan mayores beneficios económicos para las personas asociadas y su entorno (Castañeda et al., 2012, p. 14).

Es clara entonces la relación de doble vía: acciones que mejoran la situación económica de la organización favorecen el sentido de pertenencia del asociado, porque no son objetivos antagónicos. En la medida en que la ganancia económica sea justa, suficiente y equitativamente distribuida, la filosofía empresarial solidaria puede convertirse en el modelo preferido por la gente, tal como lo promueve la Alianza Cooperativa Internacional (ACI).

$\mathrm{Al}$ respecto, se trae como argumento a favor de la no contradicción entre los excedentes económicos y la conciencia social la perspectiva de Razeto (1999) de incluir la solidaridad en todos los momentos del ciclo económico, con lo cual se encuentra una conexión con prácticas de responsabilidad social empresarial. En el sentido que lo nombra Estrada, Monroy y Ramírez (2005), "la responsabilidad de una empresa implica el compromiso de ocuparse de su papel en la sociedad como: productora, empleadora, vendedora, cliente y miembro de la ciudadanía, de manera responsable y sostenible".

Pero esta armonización de objetivos económicos y sociales demanda una revisión de los enfoques y las prácticas de gestión empresarial. Herrera, Louge y Ripa (2012) hacen un análisis comparativo de la evolución de empresas capitalistas y solidarias, señalando el hecho de que los modelos de gestión cooperativa y solidaria no han evolucionado teóricamente, mientras que la teoría administrativa propia de las corporaciones ha presentado un mayor número de cambios paradigmáticos. También indican que la evolución en la teoría de la administración ha incorporado conceptos que ya estaban en la filosofía cooperativista; es el caso de la gestión de lo que las empresas denominan recurso humano, que en la economía de la solidaridad es el eje fundamental, al hacer del trabajo la centralidad, "poniéndose de este modo el hombre y su actividad por sobre las cosas y su valor monetario" (Razeto, 2002, p. 39).

La empresa capitalista avanza en modelos empresariales más horizontales, motivacionales, con estructuras flexibles, mientras que algunas empresas asociativas mantienen esquemas de gestión vertical, e incluso se incorporan prácticas de gestión organizacional capi- 
talistas del siglo $\mathrm{xx}$, ya en desuso en la empresa tradicional. De otro lado, algunas oEs son tan informales en sus estructuras que no alcanzan a convertirse en una unidad productiva como tal.

\section{Posición competitiva}

La mayoría de las oes tienen un mercado micro, delimitado por la capacidad productiva, operativa y financiera de la entidad. Teniendo en cuenta lo dicho antes, se destaca que el mercado del modelo asociativo generalmente no sobrepasa las fronteras más próximas, y una de las limitantes que expresan sus asociados es la falta de preparación técnica y empresarial para la competencia o coopetencia (Nalebuff y Brandenburguer, 1996, citados en Silva, 2013), es decir, un sistema integrado conformado por las diferentes oEs, que en conjunto les permita insertarse en el mercado como un actor protagónico.

En esta perspectiva, el estudio de Arcas y Hernán$\operatorname{dez}$ (2013) señala las ventajas que ha generado el crecimiento de las cooperativas agroalimentarias españolas en su competitividad y rentabilidad. Algunas fórmulas de crecimiento interesantes son presentadas en este último estudio, fundamentadas en la fusión y la participación en conjunto con otras organizaciones; en general, se trata de una propuesta hacia una integración entre cooperativas para obtener ventajas financieras que añaden puntos a su competitividad. Sin embargo, se alude también al mayor endeudamiento y la solvencia más reducida que presentan las cooperativas más grandes, como consecuencia de mayores necesidades de financiamiento.

Siguiendo este último punto, Iturroz y Martín (2013) indican que el tamaño de la cooperativa no necesariamente determina su éxito; es decir, no hay un tamaño específico de empresa que muestre una mejor situación de forma recurrente, aunque ellos declaran que es más sencillo establecer control en formas simples de organización con pocos socios y socias, por lo menos, al principio. Estos contrastes sobre la dimensión óptima de las cooperativas y, en general, de las organizaciones de economía solidaria, pueden constituirse en un reto para investigadores cuyo trabajo puede ayudar a definir mejor los límites dimensionales en función del tipo de inserción en la dinámica económica que se pretende hacer.

En adición a la idea anterior, algunos autores resaltan como fundamental considerar que es favorable contar con un gran número de miembros. Andrade (2005) aporta indicando que "en la medida que se aumente el número de miembros se puede pensar en avanzar también en la forma organizativa que dé mejor resultado" ( $\mathrm{p}$. 7). Para la definición de tal esquema, resultan crucialesla democracia y gobernabilidad de las oes, y una acertada decisión funcional. Esta idea de crecimiento se ve limitada en pequeñas organizaciones porque se percibe que la ampliación de los asociados puede ir en detrimento de los beneficios individuales recibidos, generando un cerramiento de la entidad a otras personas y un debilitamiento progresivo porque no dispone de otras ideas, capacidades y recursos que provienen de nuevos asociados y relaciones con el entorno.

Un segundo aspecto vinculado con la posición competitiva es la capacidad de respuesta a la demanda del mercado. Uno de los puntos por resaltar de las empresas asociativas pequeñas es la baja continuidad en la producción, que no permite ofertar sosteniblemente a mercados que demandan producción masiva, aunque la producción a gran escala también debe ser revisada, pues el agotamiento de los recursos naturales no es coherente con la apuesta solidaria.

Con base en esto, Murillo (2004) cita a Esteva (1997), haciendo referencia a que se ha concebido la sostenibilidad como una estrategia para mantener el ritmo de crecimiento económico centrado en la explotación de los recursos naturales, y no para apoyar el florecimiento y la perduración de una vida social y natural infinitamente diversa (Murillo, 2004, p. 28). Además, añade: “[...] (de ahí que se hable de mercados de agua, de prohibiciones, servicios ambientales, vedas y castigos para quien contamina más, sin un punto de vista integral y verdaderamente ecologista) y deja de lado otras ganancias, es decir, metas, como la equidad y el cuidado del ambiente" (Murillo, 2004, p. 639).

Las organizaciones solidarias han encontrado respuestas a este dilema ético y productivo. Se hace referencia a experiencias que han sabido valerse de los mismos preceptos del pensamiento solidario usándolo como estrategia competitiva mediante el consumo responsable; tal es el caso de la tienda de comercio justo ColyFlor, existente en Medellín, lo que podría llegar a ser una estrategia, con el fin de aprovechar y promover el pensamiento solidario para competir en el mercado. Se han iniciado esfuerzos para concebir de manera diferente el papel que desempeñan las organizaciones de economía solidaria (oEs) en el mercado y las potencialidades y amenazas del sistema cooperativo (Castillo et al., 2013). La necesidad de convertirse en 
organizaciones competitivas no necesariamente tiene que erosionar el compromiso social; esto tiene que ser previsto por las oes para que ellas mismas puedan establecer estrategias de fortalecimiento.

Existen evidencias para creer que las organizaciones cooperativas pueden ser tan eficientes como las organizaciones no cooperativas en términos económicos y de sustentabilidad (Hutton, Griffiths, Sumaila y Pitcher, 2001; Kalogeras et al., 2013), detrás de lo cual subyace la necesidad de una reflexión más profunda acerca de los factores culturales y sociales que limitan el desempeño empresarial.

Finalmente, resulta pertinente resaltar que la falta de competitividad de las organizaciones del sector cooperativo y solidario no sólo proviene de su interior, y que tampoco todas las soluciones pueden salir de ellas. En algunos casos, como el de los pescadores de Taganga, participantes del estudio, se ve más difícil crear una cooperativa que otro tipo de asociaciones, por sus requerimientos legales y excesiva supervisión, que afecta en forma diferenciada a las cooperativas y organizaciones solidarias en las zonas costeras y marginadas, donde el contexto socioeconómico impone más obstáculos a los ya inherentes al proceso de iniciar una nueva organización y mantenerla, siempre en desventaja en comparación con empresas que surgen en el medio urbano y capitalizado.

\section{Economía solidaria y cooperativismo en la pesca artesanal colombiana}

Las tensiones que la sociedad colombiana vive en la actualidad transcurren en medio de una expectativa de negociación del conflicto armado, con la aspiración del logro de la paz, mientras que en el campo del desarrollo económico se implementan tratados de libre comercio, con Estados Unidos, Canadá y otros países, políticas que se complementan con una "ascendente inserción en los mercados internacionales, sobre la base de productos basados en mano de obra no calificada y recursos naturales, es decir, ventajas comparativas estáticas" (Torres, 2012, p. 57).

En medio de las controversias que suscita la coyuntura política y económica del país, se encuentra el ciudadano común, que en términos generales y discursivamente se identifica con el sector de la economía solidaria al definir la paz como "[...] condiciones de vida digna generadoras de paz relativas a la equidad, la justicia social, la superación de la miseria y la libertad" (Sacipa, Tovar, Sarmiento, Gómez y Suárez, 2013), pero es sobre estos mismos ciudadanos que recaen las políticas neoliberales impulsadas por el gobierno, cuyos resultados no se relacionan con el logro de estos objetivos popularmente demandados.

En Colombia, existen problemáticas sociales y violencias derivadas de una presencia irregular y fragmentaria del Estado colombiano en ciertas regiones, sectores y actividades económicas, que pueden enmarcarse según el análisis de Ríos, Bula y Brocate (2013) en un Estado fallido o parcialmente fallido, debido a la "pérdida del control y poder político de que adolece el Estado para con su territorio", sumado a otros fenómenos como la pobreza, la desigualdad y la desarticulación de la sociedad civil.

Prácticas de contraste evidencian la idea anteriormente expuesta; a la par de la promoción del pensamiento solidario que realizan entidades estatales, se lleva a cabo un amplio consumo de productos importados y acceso a servicios bancarios internacionales que impulsan aperturas de mercado que no son creadas con un enfoque solidario, ni las rigen principios de comercio justo o de equilibrio en la negociación para las partes. En este sentido, Mercader (2013) cita a Fajardo (2012) para recordar la necesidad de la aplicabilidad de la solidaridad, de su autenticidad, para que efectivamente sea un factor de cambio estructural en la sociedad.

Hasta el momento, el modelo capitalista imperante en el país ha resultado ineficiente para crear las condiciones que permitan el aprovechamiento social de sus beneficios, ocasionando levantamientos, principalmente en las zonas rurales, como resultado del descontento de la población. El Estado colombiano reconoce esta situación que es originada por "un abandono de décadas" (El Colombiano, 20 de agosto de 2013), lo que ahora ha llevado al ejecutivo a prometer ser más inclusivo en acciones que beneficiarán a "todo el país y no a un puñado de personas” (Perea, 2013). Es aquí donde las nuevas propuestas como la economía solidaria surgen para desahogar la presión ejercida por un modelo político y económico excluyente.

En medio de este contexto, emergen las empresas solidarias. Según cifras de Confecoop (2012), en Colombia, existen 8473 entidades entre cooperativas, mutuales, fondos de empleados y otras formas asociativas; el sector genera por lo menos 522614 empleos y cuenta con 6681877 asociados. Las organizaciones del sector solidario tienen una presencia en los 32 departamentos del país y 606 de los 1103 municipios. No obstante, el sector se constituye en un $80,4 \%$ por formas asociativas 
de tamaño denominado microempresa, mientras que sólo el $0,7 \%$ son grandes empresas; asimismo, se concentra en entidades del sector financiero (Confecoop, 2012, p. 60). Detrás de estas cifras, surge una preocupación por la debilidad de la economía solidaria en sectores dinámicos de la economía: bajos niveles de integración vertical y horizontal, así como flaquezas organizativas que impiden al sector convertirse en un referente social, económico y político en el país.

Las asociaciones de tipo cooperativo entrevistadas coinciden en que hay una falta de integración dentro del mismo sistema, que indiscutiblemente las afecta en la perspectiva empresarial, ya que el sector tiene una amplia dinámica de acción en movimiento que se refleja en sus estructuras de segundo y tercer nivel, pero su actuación fundamental es en el plano social y político. En pocas oportunidades se logran generar cadenas productivas entre organizaciones pertenecientes al mismo sector económico, o ciclos económicos solidarios que articulen la producción, la distribución, el consumo, el ahorro, el crédito; en general, que aprovechen la empatía ya construida y fortalezcan la capacidad empresarial del sector cooperativo y solidario.

\section{Estructuras empresariales y liderazgo en la pesca artesanal}

Hasta el momento se han presentado discusiones generales acerca de los liderazgos y la situación empresaria de las organizaciones solidarias. En este acápite, se profundiza en el caso de los pescadores artesanales. Se entrevistaron cooperativas de pesca artesanal en Santa Marta, Taganga y Pueblo Viejo.

\section{Factores productivos y comerciales}

La situación de los pescadores de Santa Marta, en el Caribe colombiano, se diferencia de la de otras oes por las particularidades ambientales y socioculturales en las que se realiza la actividad pesquera. A diferencia de otras cooperativas, las de pesca tienen que enfrentar la incertidumbre de la captura diaria, que depende del mar, menos manipulable que la tierra. El factor ambiental se vuelve prioridad para los pescadores costeros que trabajan en medio de los residuos del sistema industrial cuyos desechos vertidos al mar son mayores que los que pudieran producir los pescadores artesanales.

Las asociaciones de pesca artesanal estudiadas, al igual que otras en diferentes partes del mundo, generan una producción mínima que las mantiene en un nivel de subsistencia. La falta de consistencia en la producción, ocasionada por el deterioro ambiental y por el debilitamiento del sistema pesquero artesanal y social en Santa Marta, ha impedido que se avance a niveles más estructurados. A pesar de una larga tradición pesquera en la zona costera donde se ubican las organizaciones de la investigación, las estructuras son simples e informales.

Según las entrevistas, el tema de insertarse en el mercado no ha sido tratado, debido a que no existen las condiciones para ofrecer constantemente productos de calidad fuera de los límites locales, sus clientes son principalmente los habitantes cercanos; algunos esfuerzos anteriores en esta perspectiva no tuvieron el impacto esperado. Asimismo, la infraestructura pesquera artesanal de las cooperativas estudiadas es escasa; no se cuenta con embarcaciones y equipo mínimo para la actividad. En el medio pesquero asociativo surgen pocas estrategias de mejoramiento debido a múltiples factores que se relacionan con la incertidumbre productiva, el bajo nivel de formación y las pocas ventajas tecnológicas. Sin embargo, algunas acciones se han realizado para fortalecer la cadena productiva. Una interesante alternativa ha sido la integración de restaurantes que son manejados por mujeres, lo que, siguiendo a Castañeda et al. (2012), significa un indicador de responsabilidad social y "potencial de la organización para otorgar un mayor valor comercial a su captura (valor agregado)" (p. 15). También existen propuestas alrededor del turismo y otras formas de agregar valor al producto fresco, que favorecen la resolución de problemas ambientales, como la aparición de nuevas especies depredadoras que pudieran ser aprovechadas para producción; tal es el caso del pez león, cuya presencia reduce los niveles de captura de otras especies, pero que también ofrece una nueva oportunidad de comercialización.

Además de lo planteado, existen necesidades básicas insatisfechas en el contexto en el que los pescadores artesanales llevan a cabo su emprendimiento solidario. Algunas comunidades no cuentan con los servicios básicos de agua potable, ni drenaje; otras no cuentan con las vías de acceso adecuadas, aparte de verse afectadas por la actividad de las empresas industriales con las que tienen que compartir los recursos del mar. Con tales condiciones, es difícil pensar en un sistema productivo que vaya más allá de la subsistencia diaria; por ello, a veces, la actividad pesquera se realiza para suplir las necesidades vitales diarias, más que para estructurar un sistema productivo. 
Aunado a lo anterior, las comunidades pesqueras habitan en medio de otras problemáticas, como la presencia de actores armados ilegales de diferente naturaleza, la falta de oportunidades y el conflicto social que vive Colombia. La situación se vuelve más grave con la baja disponibilidad de peces, atribuida a cuestiones ambientales. Los pescadores declaran sus zonas de pesca impactadas por las empresas carboníferas, la expansión portuaria y de las carreteras, por los sistemas de drenaje mal manejados, dragados, y por privatizaciones implementadas por políticas gubernamentales. Se les suma la contaminación del mar y de las zonas de pesca, la cual proviene de fuentes difusas (Aguilar, Villanueva, Guzmán y Vázquez, 2006).

Otro aspecto por considerar es el desplazamiento originado por la expansión portuaria, que ha empujado a algunos pescadores a abandonar sus localidades $\mathrm{y}$, a veces, a cambiar de actividad, al quedar más alejados del mar. Mientras tanto, aún perviven poblaciones que sienten un gran arraigo por las zonas costeras en las que han habitado sus antepasados, estableciendo un vínculo místico con la actividad pesquera artesanal $y$, por otra parte, un gobierno que promueve la expansión urbana e industrial, favoreciendo la inversión extranjera, lo que deja a las comunidades desprotegidas.

En otro orden de ideas, se destaca que algunos pescadores prefieren no constituirse como cooperativa por diferentes motivos, como las exigencias fiscales y la excesiva supervisión; estas pueden ser algunas de las causas del fracaso de planes de apoyo estatal realizados anteriormente. En el ordenamiento jurídico colombiano se identifican normas que establecen barreras al desempeño de las actividades productivas que pueden realizar las cooperativas, situación que se intensificó desde finales del siglo $\mathrm{xx}$, principalmente como resultado de las previsiones y ajustes producidos en la Ley 454 de 1998 (Zabala, 2013).

\section{El liderazgo asociativo y solidario}

Es difícil identificar un modelo predominante de liderazgo en las asociaciones entrevistadas. Algunas organizaciones establecen sus líderes formales, pero también se presenta el caso de que quien dirige los procesos es diferente a quien representa, y el reconocimiento de liderazgo lo tiene otra persona entre los asociados. La mezcla entre formas organizativas básicas y tomas de decisiones complejas es la característica común a las entidades.
En las entrevistas realizadas a oEs colombianas, se indica que para alcanzar el éxito se necesitan liderazgos con un fuerte compromiso social, visión empresarial, eficiencia, confianza al interior y al exterior de la organización, renovación generacional y planeación. En todos los casos que fueron entrevistados se encuentran líderes potenciales identificados por los miembros, pero en la mayoría, el directivo de turno no es reconocido como la mejor opción para la dirección; tal vez esto se deba a la necesaria rotación del poder en la organización y al tamaño, que admite que las elecciones se fundamenten en el comportamiento de la persona, más que en sus capacidades técnicas e intelectuales.

Existen limitaciones para el crecimiento y fortalecimiento empresarial que se originan en estas estructuras solidarias relacionadas con los intereses de sus miembros, así como otras que provienen del contexto en el que se desenvuelven. Según algunas personas entrevistadas, los obstáculos ya señalados se asocian con un devenir poco solidario en las organizaciones. Se crea una conciencia solidaria en el momento fundacional, pero generalmente se ve reducida con el paso del tiempo cuando surge un pensamiento más individualista y utilitario en términos económicos, lo cual puede estar relacionado con la tensión que se produce entre los intereses individuales y colectivos, en la que los segundos parecen ser anulados por el interés común, pero que finalmente afloran porque hacen parte de las expectativas de los sujetos en su interacción social y económica.

Retomando una discusión planteada en el primer segmento de este documento, acerca de la pertinencia de un agente externo que incorpore capacidades técnicas a la organización, el trabajo con las cooperativas pesqueras indica que a pesar de las evidencias de éxito de modelos de dirección muy rígidos en algunas organizaciones, en donde un líder externo recurre a estrategias poco flexibles (Scarnatti, 2002) por un tiempo relativamente corto, es profundamente difícil que este método funcione en un medio como el de la pesca artesanal colombiana donde el hilo del orden social es muy frágil y en el que los modelos no democráticos son poco atractivos.

De acuerdo con las formas de pensar predominantes entre pescadores, puede ser que un líder que priorice las tareas y no a la gente llegue a resultar poco efectivo en este contexto. De igual manera, es difícil incluir personas externas en un entorno tan cerrado y que no surjan problemas que obstaculicen el proceso. Los liderazgos ejercidos dentro de estas asociaciones 
se fundamentan en las relaciones sociales preexistentes. Por ello se hace mucho más trascendente encontrar fórmulas de un protagonismo que haga operativas las decisiones a través de normas, instrucciones, procesos $y$ criterios que generen una cultura organizativa definida, para evolucionar la forma y el contenido (Andrade, 2005) según necesidades, mientras que también se incorporan nuevos estilos de liderazgo, por ejemplo pasar del líder carismático al líder funcional.

\section{Rol del Estado en la promoción de la empresarialidad solidaria}

Los pescadores entrevistados manifiestan la desatención por parte del gobierno; han empezado a mirar a las universidades, porque les resulta una opción en mejoras organizativas, productivas y comunitarias, pero el apoyo del Estado sigue siendo fundamental, y su aporte puede surtir efectos positivos en la situación social y productiva de las zonas pesqueras, acompañado de políticas efectivas de educación formal y técnica, contextualizada para adultos. La falta de atención provoca que los pescadores tengan que ser responsables de crear condiciones favorables para ellos mismos sin contar con los medios para ello, además de estar en un entorno adverso para posicionar iniciativas económicas viables de tipo solidario y comunitario.

Estas condiciones precarias van cambiando de manera muy lenta. Los problemas sociales están recibiendo más atención que en años anteriores, aunque el aspecto productivo pesquero artesanal se ha mantenido al margen durante un largo tiempo. Otras actividades, como la piscicultura, han fracasado en el pasado por falta de apoyo tanto formativo como técnico, mientras que en algunos casos existe una excesiva ayuda financiera por parte del Estado (Castillo et al., 2013) que ha beneficiado proyectos individuales o de agentes con capacidad propia para insertarse en el mercado, con lo cual se aporta poco para el avance del desarrollo de las economías locales.

Acompañando a estas prácticas de intervención estatal puntuales, también se produce un desinterés por parte del Estado, que privilegia la competitividad empujando a organizaciones débiles al fracaso. En la priorización de la apertura a los mercados internacionales, el Estado carece de estrategias para proteger a las cooperativas y a las Pymes frente al Tratado de Libre Comercio, dejando esta responsabilidad para las mismas organizaciones. En esta perspectiva, se necesita revisar el rol del Estado; como lo manifiesta Murillo (2004) citando a Funes (1985):

[el Estado] no es el protagonista del desarrollo, sino solamente un instrumento de los sectores sociales que, siendo los protagonistas del desarrollo, dirimen constantemente en el interior del aparato de Estado y fuera de él cuáles son los intereses y proyectos en función de los que la sociedad modela sus destinos.

Queda asentado, entonces, que el papel más importante es el de la ciudadanía colectivamente organizada, cuyas necesidades y aspiraciones sociales orientan la acción estatal; en relación con esto, las estrategias que surjan dentro de las comunidades en las que se lleva a cabo la pesca deben dialogar con actores que puedan ejercer un sistema de acompañamiento (Lussier y Achua, 2008).

Retomando el tema productivo mencionado anteriormente, se evidencia que la incertidumbre en los volúmenes de pesca debe ser atendida en conjunto por el gobierno y los pescadores artesanales. De acuerdo con Lluch (2006, p. 268), es tarea del ejecutivo establecer los volúmenes de captura permisibles, regular el conjunto de instrumentos, artes, equipos, personal y técnicas pesqueras, el número de embarcaciones y sus características aplicables a la captura de determinada especie, fijar la época, talla o peso mínimos de los especímenes susceptibles de consumo y proponer las normas para su manejo, conservación y traslado, ya que la actividad pesquera tiene un efecto directo en las condiciones ambientales de las zonas de extracción de las especies marinas.

En un entorno de presiones ambientales, se deben considerar los aspectos que permitan reducir el deterioro ocasionado, tanto por la pesca artesanal como por la pesca industrial. Hutton et al. (2001) señalan cómo modelos competitivos en la pesca pueden llevar a una sobreexplotación del recurso, fundamentados en modelos de producción a gran escala, que no cumplen criterios de sostenibilidad ambiental.

Esto demanda una fuerte preparación por parte de quienes realizan la actividad pesquera cooperativa con el objetivo de crear una conciencia de cuidado ambiental, lo cual puede resultar más sencillo si se tiene en cuenta que la mayoría de las asociaciones de pescadores están conformadas por la misma gente que habita la zona de pesca; aunque también se debe tener presente la contaminación que surge de los asentamientos humanos. El compromiso debe ser al interior y al 
exterior de las comunidades, de manera que los habitantes puedan reapropiarse del entorno con prácticas más ecológicas.

Es indispensable la integración del Estado en el desarrollo estructural de la pesca para equilibrar los obstáculos originados por la problemática social y ambiental. Sobre este particular, Aguilar, Villanueva, Guzmán y Vázquez (2006) señalan que los estudios pesqueros y acuícolas deben adoptar el enfoque ecosistémico que incluye la dimensión antropológica, la socioeconómica y la ambiental, y que deben hacerlo en forma integral e interdisciplinaria a lo largo de la cadena productiva, desde su extracción o cultivo, hasta su comercialización.

\section{Conclusiones}

Las organizaciones solidarias pueden encontrar el éxito (entendido como cumplimiento de sus objetivos económicos y sociales) haciendo del discurso asociativo su ventaja comparativa y competitiva. No hay que descartar el liderazgo como factor clave de éxito, un estilo gerencial que combina reconocimiento entre los asociados y capacidad para la toma de decisiones empresariales. Es importante señalar la necesidad de generar nuevos protagonismos, que se adhieren a la ética solidaria y que a su vez aportan capacidad técnica para el desarrollo de la organización.

Sin embargo, el tema de la estructura organizacional es determinante; el tamaño y la gestión empresarial deben ser funcionales al entorno económico en el que se desenvuelve la entidad solidaria. El reto también es trabajar en la integración de un sistema fuerte que equilibre lo social con lo empresarial, para poder hacer frente a la agresiva competencia ejercida por las corporaciones. Para lograrlo resulta indispensable la participación del Estado, cuya responsabilidad recae en brindar apoyo a ciudadanos comprometidos con ideas productivas viables.

Ahora, en cuanto a la pesca artesanal de las zonas de Pueblo Viejo, Santa Marta y Taganga, específicamente, tiene que enfrentar condiciones desfavorables para obtener una estructura productiva eficiente. La cuestión social y ambiental debe ser tratada de manera integral por el gobierno, en conjunto con las mismas comunidades pesqueras. En las zonas de pesca abundan problemas que pueden ser abordados desde la investigación multidisciplinaria. Aspectos propios del desarrollo social, como el combate a la pobreza, el acceso a la educación y el fortalecimiento de los sistemas productivos se pueden entrelazar con estudios de monitoreo marino en los que se pueda conocer el estado del recurso pesquero y sus condiciones en relación con los niveles de desechos que percibe el mar.

El papel del Estado es fundamental para el desarrollo social y organizativo de la pesca en el Magdalena. Las precarias condiciones de las zonas en que se desarrolla la pesca no favorecen su avance productivo. Igualmente generan tensiones sociales y descontento de las comunidades hacia las instituciones, lo que se convierte en un obstáculo para los planes estatales que pudieran llevar al sistema pesquero artesanal a establecerse competitivamente dentro del mercado con un enfoque solidario.

Por otra parte, también es necesario que el avance provenga de acciones impulsadas desde adentro de los grupos de pescadores, para superar obstáculos identificados: falta de integración con el sector solidario en la perspectiva productiva; debilidad en la vivencia de los valores cooperativos; informalidad con la que operan; mecanismos democráticos sin funcionalidad para la toma de decisiones empresariales; solo presencia de liderazgos carismáticos; falta de competitividad.

Un punto de trabajo es el diseño de modelos cooperativos en las zonas de pesca artesanal que sean sostenibles desde el punto de vista ambiental, ya que el éxito de la empresa asociativa está ligado directamente con el uso responsable de los recursos naturales.

Finalmente, los habitantes de las zonas pesqueras y los mismos pescadores necesitan crear una conciencia ecológica que se integre con las acciones del gobierno para reducir el deterioro ambiental y que pueda servir de pauta para el futuro de las generaciones. El relevo generacional en las organizaciones y el apoyo educativo a jóvenes puede ser un buen comienzo.

\section{Referencias}

Aguilar, I. A., Villanueva, F. S., Guzmán, A. P. y Vázquez, B. A. (2006). La contaminación del agua como una externalidad para la producción pesquera y acuícola. En Pesca, acuicultura e investigación en México. México: Centro de Estudios para el Desarrollo Rural Sustentable y la Soberanía Alimentaria, Cámara de Diputados.

Andrade, S. (2005). Diseño y arquitectura organizativa en la globalización. Administración y Organizaciones, 15(8), 71-92.

Arcas, N. y Hernández, M. (2013). Tamaño y competitividad: casos destacados de cooperativas agroalimentarias españolas. Mediterráneo Económico, 24, 205-229. 
Castañeda, N., Guido, S. y Medina, F. (2012). Cooperativas exitosas en Sinaloa: lecciones para aprender y compartir. México: The Walton Family Foundation; Conselva, Costas y Comunidades; Universidad Autónoma de Sinaloa y Comisión Nacional de Pesca y Acuacultura. Disponible en http://www.conapesca.sagarpa.gob.mx/ work/sites/cona/dgof/publicaciones/CooperativasPesquerasExitosas.pdf

Castillo, D., Dávila, R., Sánchez, C., Rodríguez, N., Rodríguez, D. y Monsalve, A. (2013). Globalización, internacionalización y sector cooperativo en Colombia: una aproximación teórica. En Instituto de Estudios del Ministerio Público, IEMP (Ed.), Cooperativismo e internacionalización: Vol. 1. Retos y perspectivas frente a los TLC. Bogotá: Unidad Administrativa Especial de Organizaciones Solidarias.

Confecoop. (2012). Desempeño del sector cooperativo 2012. Disponible en http://cenicoop.coop/images/archivospdf/Informe_2012.pdf

El Colombiano. (20 de agosto de 2013). "El paro es el resultado de un abandono de décadas": presidente Santos. Disponible en http://www.elcolombiano.com/historico/paro_agrario_el_paro_es_el_resultado_de_un_ abandono_de_decadas_dijo_santos-BCEC_257745

Estrada, R., Monroy, G. y Ramírez, H. (2005). Ética, responsabilidad social y desarrollo sustentable en las organizaciones. Administración y Organizaciones, 15(8), 27-45.

Fiedler, F. (1967). A Theory of Leadership Effectiveness. New York: McGraw-Hill.

Fortier, R. (2011). Liderazgo cooperativo vs. Liderazgo corporativo: ¿Cómo somos diferentes, cómo somos iguales? En Retos y oportunidades de liderazgo cooperativo y seguros mutuos. XIX Conferencia Anual de ICMIF/ Américas. Costa Rica.

Herrera, A., Louge, R. y Ripa, M. (2012). Las empresas cooperativas construyen un mundo mejor. En Propuestas del cooperativismo: mensaje, ponencias y debates del Congreso Argentino de las Cooperativas 2012. Editora Cooperativa Limitada.

Hutton, T., Griffiths, M. H., Sumaila, U. R. y Pitcher, T. J. (2001). Cooperative versus Non-Cooperative Management of Shared Linefish Stocks in South Africa: An Assessment of Alternative Management Strategies for Geelbek (Atractoscion aequidens): Fisheries Research, 51(1), 53-68.

Iturroz, J. y Martín, S. (2013). El tamaño como elemento determinante de la insolvencia en las sociedades cooperativas: Estudio a partir de los procesos concursales. Revista de Estudios Cooperativos, 11, 90-107.

Kalogeras, N., Pennings, M., Benos, T. y Doumpos, M. (2013). Which Cooperative Ownership Model Performs Better? A Financial-Decision Aid Approach. Agribusiness, 29(1), 80-95.
Karsten, M. (2009). Coaching an Effective Leadership Intervention. Nursing Clinics of North America, 45(1), 39-48.

Lluch, D. (2006). Políticas y obligaciones del Estado relativas a la investigación científica y tecnológica, pública y privada. En Centro de Estudios para el Desarrollo Rural Sustentable y la Soberanía Alimentaria, CEDRSSA (Ed.), Pesca, acuacultura e investigación en México. México: Comisión de Pesca.

Lussier, R. y Achua, C. (2008). Liderazgo: teoría, aplicación $y$ desarrollo de habilidades. México: Cengage Learning Editores.

Mercader, V. (2013). Reflexión integral sobre la ética de las cooperativas. En J. Álvarez (Ed.), Cooperativismo e internacionalización: Vol. 2. Condiciones y lineamientos para su desarrollo en Colombia. Bogotá: Unidad Administrativa Especial de Organizaciones Solidarias.

Murillo, D. (2004). Falacias del desarrollo sustentable: una crítica desde la metamorfosis conceptual. Economía, sociedad y territorio, IV(16), 635-656. Disponible en http:// est.cmq.edu.mx/index.php/est/article/view/406/798

Perea, A. (2013). Pacto agrario en Colombia está avanzando. El Universal. Disponible en http://www.eluniversal. com.co/economica/pacto-agrario-en-colombia-estaavanzando-ruben-dario-lizarralde-140063

Razeto, L. (1999). La economía solidaria: concepto, realidad y proyecto. Revista Persona y Sociedad, XIII(2). Disponible en http://www.luisrazeto.net/content/la-econom\% C3\%ADa-solidaria-concepto-realidad-y-proyecto

Razeto, L. (Febrero, 2002). Economía de solidaridad y profundización de la democracia para una nueva civilización. Ponencia presentada en el II Foro Social Mundial, Porto Alegre, Brasil. Disponible en http:// www.luisrazeto.net/content/econom $\% \mathrm{C} 3 \% \mathrm{ADa}$-desolidaridad-y-profundizaci\%C3\%B3n-de-la-democracia-para-una-nueva-civilizaci\%C3\%B3n

Ríos, S. J., Bula, E. G. y Brocate, P. R. (2013). Estado, estado de derecho y violencia armada en Colombia. Revista de Paz y Conflictos, 6, 6-31 Disponible en http://www.ugr. es/ revpaz/numeros/rpc_n6_2013_completo.pdf

Sacipa, R. S., Tovar, G. C., Sarmiento, L., Gómez, A. y Suárez, M. P. (2013). La psicología política en Colombia. Cahiers Psychologie Politique, 23. Recuperado de http:// lodel.irevues.inist.fr/cahierspsychologiepolitique/index.php?id=2559.

Scarnatti, J. (2012). The Godfather Theory of Management: An Exercise in Power and Control. Management Decision, 40(9), 834-841.

Segura-Aguilar, R., Rodríguez-Van Dyck, S. y Weaver, A. H. (2009). Guía práctica para sociedades cooperativas de producción pesquera. México: Sociedad de Historia Natural Niparajá A. C. y RARE inspirando la conservación A. C., B. C. s. 
Silva, A. (2013). Algunas pautas de gestión para la coopetitividad. En J. Álvarez (Ed.), Cooperativismo e internacionalización. Vol 2. Condiciones y lineamientos para su desarrollo en Colombia (pp. 161-177). Bogotá: Unidad Administrativa Especial de Organizaciones Solidarias.

Sommers, G. (2006). El cooperativismo y las cooperativas pesqueras del Soconusco. Antecedentes, desarrollo y praxis. Disponible en http://www.eumed.net/ librosgratis/2012a/1154/cooperativismo_y_cooperaticas_pesqueras.html

Torres, D. (2012). Veinte años de comercio exterior en Colombia. Revista Expeditio, 12, 41-59. Disponible en http://revistas.utadeo.edu.co/index.php/EXP/article/ view/759/767
Weber, M. (1920/2007). Sociología del poder: los tipos de dominación. Madrid: Alianza.

Whitmore, J. (1992). Coaching for Performance. Boston: Nicholas Brealey Publishing Limited.

Zabala, H. (2013). Definiendo el problema: los obstáculos a la internacionalización cooperativa. Cooperativismo e internacionalización. Vol. 2. Condiciones y lineamientos para su desarrollo en Colombia (pp. 57-91). Bogotá: Unidad Administrativa Especial de Organizaciones Solidarias. 